\title{
UPDATES IN MANAGEMENT OF POSTOPERATIVE ENDOPHTHALMITIS
}

\author{
Farouk, M. ${ }^{(*)}$ \\ Ophthalmology dept.., Faculty of Medicine, Sohag Univ., Sohag, Egypt \\ *E-mail: farouk.eye@gmail.com
}

\begin{abstract}
Endophthalmitis is one of the most devastating complications of intraocular surgeries. The interest in the management of endophthalmitis pushed most of the researchers to find new more efficient protocols for proper management of this sight threatening complications. The aim of this review is to discuss the recent advances in management of endophthalmitis including prevention, diagnosis and treatment.
\end{abstract}

Keywords: Endophthalmitis; cataract, Intracameral cefuroxime, Moxifloxacin; Polymerase chain reaction, Vitrectomy

\section{Introduction}

Cataract surgery represents the most common intraocular surgery in ophthalmic field. Endophthalmitis is one of the most devastating complications of intraocular surgeries, fortunately it is rare. Most of the studies about postoperative endophthalmitis are centered on cataract surgery [1]. The aging population is increasing all over the world with subsequent increase in the number of cataract operations, rendering post-cataract surgery endophthalmitis a public health concern. The interest in the management of endophthalmitis pushed most of the researchers to find new more efficient protocols in prevention, diagnosis and treatment of this dangerous complication [2].

\section{Updates in Prevention of Endophthalmitis}

To determine the most effective prophylactic regimen, the ESCRS study was conducted. It was a multicenter clinical trial on 16,603 patients to evaluate prospectively the preventive effect of intracameral cefuroxime and/or perioperative eye drops $(0.3 \%$ levofloxacin $)$ on the incidence of postoperative endophthalmitis after cataract surgery. The results concl- uded that intracameral cefuroxime administered at the time of surgery significantly reduced the incidence of postoperative endophthalmitis [3]. So, intracameral antibiotics became the hottest topic in the research field for prevention of postoperative endophthalmitis. Cefuroxime, Vancomycin and Moxifloxacin were used with rising questions about the antibiotic type, 
safety profile, and potential complications [3]. Intracameral cefuroxime has been proven by other studies (e.g. the landmark European Society of Cataract and Refractive Surgeons trial) to be well tolerated and effective in the prevention of endophthalmitis after cataract surgery. The intravenous form can be used as an off-label drug, but it requires two step dilutions to avoid concentration errors. A special form was designed for ophthalmic use for intracameral injection (Aprokam ${ }^{\circledR}$, intracameral cefuroxime) and has been proven in the European area for prevention of endophthalmitis by decreasing its incidence by 5 to 9 folds [4]. At increased dose, cefuroxime toxicity can result in toxic anterior segment syndrome (TASS) and posterior segment inflamemation with fibrin formation, corneal edema,

\section{Updates in Diagnosis}

The clinical diagnosis is the most important tool in diagnosis of Endophthalmitis and further supported by imaging and special laboratory tests. Bacterial culture of obtained vitreous sample is a classic method of diagnosis by unfortunately it is negative in $30 \%$ of cases [8]. B-Scan ultrasonography is another important tool for diagnosis [5]. Polymerase chain reaction (PCR) is an alternative molecular diagnostic tool which can augment

\section{Updates in treatment}

Endophthalmitis Vitrectomy Study (EVS) was conducted between 1990 and 1994, trying to find a role for decision making in treatment of endophthalmitis and proved that intravitreal antibiotics have an equal effect to core vitrectomy in management of cases of postoperative endopthalmitis with visual acuity of hand motion or better [11]. But, should we still follow the endophthalmitis vitrectomy study more than two decades after its publication? Along this period a lot of advances in the technique of vitrectomy improved its outcomes and raise the elevated intraocular pressure, serous macular detachment, CME, hemorrhagic retinal infarction [5]. Moxifloxacin is another antibiotic which can be used intracameral to prevent postoperative endophthalmitis and proved to be effective and safe as well as cefuroxime. It can be prepared from the eye drops of Moxifloxacin 0.5\% (Vigamox) by diluting $3 \mathrm{cc}$ with $7 \mathrm{cc}$ of balanced salt solution. The prepared solution can be used for intracameral injection and for hydration of the corneal wounds [6]. The use of intracameral Vancomycin was discouraged by some reports due to the risk of Hemorrhagic occlusive retinal vasculitis and to avoid the rise up of resistant organisms as it was known as the antibiotic of choice for Methicillin-resistant Staphylococcus aureus (MRSA) [7].

and improve pathogen detection. It provides quantification of the organism load and can detect multiple organisms at once. A more advanced technique of PCR is the deep sequencing techniques. This technique can allow highly sensitive detection of any DNA-based life form the vitreous or aqueous sample. So, it can improve the detection of traditional organisms and also identify organisms not previously associated with endophthalmitis [10].

value of vitrectomy in management of endophthalmitis [12]. Complete and Early Vitrectomy for Endophthalmitis (CEVE) is a new emerging protocol as an alternative to the Endophthalmitis Vitrectomy Study. Currently most of the ophthalmic practitioners prefer to manage postoperative endophthalmitis by early complete vitrectomy due to several reasons. Firstly, early intervention will protect the intraocular tissues from the toxic effect of infections and development of further complications as retinal detachment. On the other hand, complete vitrectomy (rather 
than core vitrectomy) will decrease the load of organisms inside the eye to allow good control of infection and clearance of the vitreous cavity from these opacities.
Moreover, most of ophthalmologists now perform vitrectomy for management of endophthalmitis regardless of the visual acuity [13].

\section{Conclusion}

Endophthalmitis is one of the most devastating complications of intraocular surgeries. The most recent advances in management of endophthalmitis include the preventive effect of intracameral cefuroxime, the use of Polymerase chain reaction (PCR) as an alternative molecular diagnostic tool for improving pathogen detection and the role of complete and early Vitrectomy for Endophthalmitis (CEVE) as a new emerging protocol which is alternative to the Endophthalmitis Vitrectomy Study.

\section{References}

1. Sandvig K., Dannevig L. Postoperative endophthalmitis: establishment and results of a national registry. J. Cataract Refract Surg. 2003; 29: 1273-1280.

2. Jabbarvand M., Hashemian H., Khodaparast M., Jouhari M., Tabatabaei A., Rezaei S. Endophthalmitis occurring after cataract surgery: outcomes of more than 480000 cataract surgeries, epidemiologic features, and risk factors. Ophthalmology 2016; 123 (2): 295-301.

3. Barry P., Seal D., Gettinby G., Lees F., Peterson M., Revie C.; ESCRS Endophthalmitis Study Group. ESCRS study of prophylaxis of postoperative endophthalmitis after cataract surgery: Preliminary report of principal results from a European multicenter study. J. Cataract Refract Surg. 2006; 32 (3):407-410.

4. Keating G. Intracameral cefuroxime: Prophylaxis of postoperative endophthalmitis after cataract surgery. Drugs 2013; 73 (2): 179-186.

5. Ng A., Tang W., Li P., Li K. Intracameral cefuroxime in the prevention of postoperative endophthalmitis: an experience from Hong Kong. Graefes Arch Clin Exp Ophthalmol 2016; 254(10):1987-1992.

6. Arshinoff S., Modabber M. Dose and administration of intracameral moxifloxacin for prophylaxis of postoperative endophthalmitis. J. Cataract Refract Surg. 2016; 42 (12): 1730-1741.

7. Witkin A., Chang D., Jumper J., Charles S., Eliott D., Hoffman R., Mamalis N., Miller K., Wykoff C. Vancomycin-Associated Hemorrhagic
Occlusive Retinal Vasculitis: Clinical Characteristics of 36 Eyes. Ophthalmology 2017; 124 (5): 583-595.

8. Hashemian H, Mirshahi R, Khodaparast M, Jabbarvand M. Post-cataract surgery endophthalmitis: Brief literature review. J. of Current Ophthalmology 2016; 28: 101-105

9. Vaziri K., Schwartz S., Kishor K., Flynn Jr H. Endophthalmitis: State of the art. Clin Ophthalmol 2015; 9: 95108.

10. Hong B., Lee C., Van Gelder R., Garg $\mathrm{S}$. Emerging techniques for pathogen discovery in endophthalmitis. Curr Opin Ophthalmol 2015; 26 (3): 221-225.

11. Endophthalmitis vitrectomy study group. Results of the endophthalmitis vitrectomy study. Arch Ophthalmol 1995; 113: 1479-1496

12. Grzybowski A., Schwartz S., Matsuura K., Ong Tone S., Arshinoff S, Ng J., et al. Endophthalmitis prophylaxis in cataract surgery: Overview of current practice patterns around the world. Curr Pharm Des 2017; 23 (4): 565573.

13. Soliman M., Gini G., Kuhn F., Iros M., Parolini B., Ozdek S., et al. International practice patterns for the management of acute postsurgical and postintravitreal injection endophthalmitis: European Vitreo-Retinal Society Endophthalmitis Study Report 1 . Ophthalmol Retina 2019; 3 (6): 461467. 